\title{
Macromolecular Nanomedicine of Glucocorticoids for the Treatment of Rheumatoid Arthritis
}

\section{Lingdong Quan*}

Arthritis and Tissue Degeneration Program, Hospital for Special Surgery, 535 East 70th Street, New York, NY 10021, USA

Glucocorticoids (GCs) are a class of steroid hormones, characterized by their ability to bind with the cortisol receptors and trigger various biological effects. They have potent anti-inflammatory and immunosuppressive properties, and therefore, following the Nobel Prize-winning discovery in 1948 by Hench and colleagues, glucocorticoids initially were widely used for Rheumatoid Arthritis (RA). However, only within several years, the classical adverse effects of glucocorticoids-including an increased prevalence of adrenal insufficiency, infection, cataracts, and secondary osteoporosis-were frequently noted, particularly at high dose being long-term used, and this markedly limited glucocorticoid use in clinic [1]. Despite the concerns limiting the enthusiasm of GCs use, it is estimated that over $50 \%$ of patients with Rheumatoid Arthritis have been treated, more or less, continuously with GCs [2]. Overall, the market for GCs is estimated as $\$ 10$ billion per year [3].

Today, the use of GCs in RA remains one of the most controversial areas of modern arthritis management. Attitudes towards glucocorticoid therapy in RA range from suspicion to widespread acceptance [4,5]. In recent years, there has been a revival of the role of glucocorticoids in the treatment of RA [6]. Most often, GCs are used in short-term to swiftly control flare, and allow the more slow acting treatments, such as Disease-Modifying Anti-Rheumatic Drugs (DMARDs) to exert their therapeutic effects. Recent studies clearly establish the fact that with lowdosage long-term treatment, glucocorticoids can substantially reduce the rate of bone and cartilage erosion progression in RA, in addition to their well-recognized anti-inflammatory and immunosuppressive properties, with short and medium-term use [7-9].

Adverse effects of glucocorticoids can be mostly attributed to their high cumulative doses. Rapid clearance and a large volume of body distribution account for low concentrations of GCs at the target sites, and necessitate frequent application at high doses. From a pharmacological perspective, incorporation of tissue specificity and alteration of GCs in vivo distribution, would serve as a feasible strategy to overcome these limitations, therefore reducing their off-target toxicities.

During past decades, a large number of GC macromolecular nanomedicines have been developed by covalently conjugating GC molecules to biocompatible polymeric carriers, via an ester linker [10-12]. Despite the diversity of polymers (i.e. dextran, chitosan and dendrimers) and the model drug of GCs (i.e. methylprednisolone, prednisolone and budesonide) used, the general design principle of these nanomedicines is very similar. For this type of nanomedicine design, one common concern is the poor in vivo stability of ester bond, due to the abundance of esterases in blood circulation. In vitro release studies with these nanomedicines displayed rapid hydrolytic kinetics, with nearly $40 \%$ of the drug being released within $12-48 \mathrm{~h}$ in PBS (pH 7.4) $[10-12]$, Therefore, from this perspective, such designs are not practically suitable for treatment of a chronic inflammatory condition, such as RA, which requires sustained drug activation kinetics.

Successful drug delivery strategies rely to a great extent on the pathophysiological conditions of target tissue. Recognition of the char- acteristics of the RA joints and synovial pathology suggests that an acid cleavable linker might be an optimal choice for design of nanomedicine. Acidosis of Synovial Fluid (SF) is a characteristic feature of inflamed joints, where the $\mathrm{pH}$ value of $\mathrm{SF}$ has been shown to be as low as 6.0 , and in some cases, even lower than $5.0[13,14]$. By linking the drug and the macromolecular carrier with an acid-cleavable bond (e.g. hydrazone bond, cis-aconityl bond, Schiff base or acetal bond), this unique pathophysiological feature can be exploited as a disease-specific drug activation mechanism, which would further define its inflammatory joint specificity. Moreover, the intracellular lysosomal compartment (pH 5.5-6) might be another activation trigger for nanomedicines with acid-sensitive linkage, since most of the polymeric conjugates are trafficked into a lysosomal compartment, after their endocytosis by activated synoviocytes. In a series of recently published articles, Wang's group successfully exploited this strategy and reported attractive treatment results, by developing a novel N-(2-hydroxypropyl)methacrylamide (HPMA)-based dexamethasone (Dex) nanomedicine [15-18] The two carbonyl groups of Dex were employed as potential conjugation sites, by linking them to the HPMA copolymer, via a hydrazone bond. An in vitro study found that HPMA-Dex nanomedicine was indeed cleavable under acidic conditions $(\mathrm{pH}=5.0)$, at a rate of $1 \%$ of the total Dex loaded per day during the entire testing period (14 days), while no significant Dex release was found in PBS (pH 6.0 and 7.4) or in rodent plasma $[15,17]$. Moreover, in an adjuvant-induced arthritis animal model, a single treatment with HPMA-Dex nanomedicine was able to provide complete and sustained resolution of the ankle joint inflammation. The treatment also resulted in structural preservation of the articular bone and cartilage [18]. In addition to the efficacy study, the impact of the structural parameters (e.g. molecular weight, drug loading, etc.) on the nanomedicines' Pharmacokinetics and Biodistribution (PK/BD) profiles has been investigated by Quan et al. [17], using the AA rat model. The increase of either molecular weight (from 14 to 40 $\mathrm{kDa}$ ) or Dex content (from 0 to $313 \mu \mathrm{mol} / \mathrm{g}$ ) facilitated the distribution of HPMA-Dex nanomedicine to the arthritic joints, presumably due to prolonged circulation half-life and enhanced inflammatory cell uptake at the sites of inflammation.

In spite of potential deleterious effects, glucocorticoids continue to be an important and highly prescribed component of the treatment regimen, for patients with Rheumatoid Arthritis. An increasing body

*Corresponding author: Lingdong Quan, Arthritis and Tissue Degeneration Program, Hospital for Special Surgery, 535 East 70th Street, New York, NY 10021, USA, Tel: 212-774-2529; E-mail: quanl@hss.edu

Received November 17, 2012; Accepted November 19, 2012; Published November 21, 2012

Citation: Quan L (2013) Macromolecular Nanomedicine of Glucocorticoids for the Treatment of Rheumatoid Arthritis. J Nanomed Nanotechol 4:e126. doi:10.4172/2157-7439.1000e126

Copyright: () 2013 Quan L. This is an open-access article distributed under the terms of the Creative Commons Attribution License, which permits unrestricted use, distribution, and reproduction in any medium, provided the original author and source are credited. 
of literature from well-designed clinical trials supports the efficacy of glucocorticoids, for both short-term symptomatic relief, and as a disease-modifying agent. To better balance the benefits and risk of adverse effects, the introduction of macromolecular nanomedicine for RA represents a promising direction, though still in its infancy, compared to the wide application of macromolecular nanomedicine in cancer treatment. The current macromolecular nanomedicines for RA are primarily designed with an activation mechanism, triggered by enzymes or an acidic environment. More detailed knowledge regarding their exact sites of activation and retention mechanism are still missing. In order to advance the development of this strategy and to accelerate the translation of the nanomedicnes into clinical application, there is a definite need for a thorough and long-term evaluation of the efficacy and toxicities for these nanomedicines in relevant animal models.

\section{References}

1. Townsend HB, Saag KG (2004) Glucocorticoid use in rheumatoid arthritis: Benefits, mechanisms, and risks. Clin Exp Rheumatol 22: S77-S82.

2. Buttgereit F, Straub RH, Wehling M, Burmester GR (2004) Glucocorticoids in the treatment of rheumatic diseases: an update on the mechanisms of action. Arthritis Rheum 50: 3408-3417.

3. Schacke H, Docke WD, Asadullah K (2002) Mechanisms involved in the side effects of glucocorticoids. Pharmacol Ther 96: 23-43.

4. Kirwan JR, Russell AS (1998) Systemic glucocorticoid treatment in rheumatoid arthritis--a debate. Scand J Rheumatol 27: 247-251.

5. Laan RF, Jansen TL, van Riel PL (1999) Glucocorticosteroids in the management of rheumatoid arthritis. Rheumatology (Oxford) 38: 6-12.

6. Bijlsma JWJ, Boers M, Saag KG, Furst DE (2003) Glucocorticoids in the treatment of early and late RA. Ann Rheum Dis 62: 1033-1037.

7. van Everdingen AA, Jacobs JW, Siewertsz Van Reesema DR, Bijlsma JW (2002) Low-dose prednisone therapy for patients with early active rheumatoid arthritis: clinical efficacy, disease-modifying properties, and side effects: a randomized, double-blind, placebo-controlled clinical trial. Ann Intern Med 136 $1-12$.
8. Kirwan JR, Bijlsma JWJ, Boers M, Shea BJ (2007) Effects of glucocorticoids on radiological progression in rheumatoid arthritis. Cochrane Database Syst Rev.

9. Jacobs JWG, Everdingen AAV, Verstappen SMM, Bijlsma JWJ (2006) Followup radiographic data on patients with rheumatoid arthritis who participated in a two-year trial of prednisone therapy or placebo. Arthritis Rheum 54: 1422-1428.

10. Penugonda S, Kumar A, Agarwal HK, Parang K, Mehvar R (2008) Synthesis and in vitro characterization of novel dextran-methylprednisolone conjugates with peptide linkers: effects of linker length on hydrolytic and enzymatic release of methylprednisolone and its peptidyl intermediates. J Pharm Sci 97: 26492664.

11. Onishi H, Saito $Y$, Sasatsu M, Machida $Y$ (2011) Kinetic analysis of in vitro and in vivo release of prednisolone from the conjugate of glycol-chitosan and succinyl-prednisolone. Int J Pharm 410: 17-22.

12. Perumal O, Khandare J, Kolhe P, Kannan S, Lieh-Lai M, et al. (2009) Effects of branching architecture and linker on the activity of hyperbranched polymerdrug conjugates. Bioconjug Chem 20: 842-846.

13. Goldie I, Nachemson A (1969) Synovial pH in rheumatoid knee-joints I. The effect of synovectomy. Acta Orthop Scandinav 40: 634-641.

14. Andersson SE, Lexmuller K, Johansson A, Ekstrom GM (1999) Tissue and intracellular $\mathrm{pH}$ in normal periarticular soft tissue and during different phases of antigen induced arthritis in the rat. J Rheumatol 26: 2018-2024.

15. Liu XM, Quan LD, Tian J, Alnouti Y, Fu K, et al. (2008) Synthesis and evaluation of a well-defined HPMA copolymer-dexamethasone conjugate for effective treatment of rheumatoid arthritis. Pharm Res 25: 2910-2919.

16. Liu XM, Quan LD, Tian J, Laquer FC, Ciborowski P, et al. (2010) The Syntheses of click PEG-dexamethasone conjugates for the treatment of rheumatoid arthritis. Biomacromolecules 11: 2621-2628.

17. Quan LD, Yuan F, Liu XM, Huang JG, Alnouti Y, et al. (2010) Pharmacokinetic and biodistribution studies of $\mathrm{N}$-(2-hydroxypropyl)methacrylamide copolymerdexamethasone conjugates in adjuvant-induced arthritis rat model. Mo Pharmaceutics 7: 1041-1049.

18. Quan LD, Purdue PE, Liu XM, Boska MD, Lele SM, et al. (2010) Development of a macromolecular prodrug for the treatment of inflammatory arthritis: mechanisms involved in arthrotropism and sustained therapeutic efficacy. Arthritis Res Ther 12: R170. 